\title{
An Overview on Recent Advances in Biosensor Technology and its Future Application
}

\author{
Anab Fatima ${ }^{1 *}$, Ishrat Younas ${ }^{2}$, Mohammad Waqar Ali ${ }^{1}$ \\ ${ }^{1}$ Department of Pharmaceutics and Pharmacy Practice, Faculty of Pharmacy,Dow College of Pharmacy,DUHS.Karachi.Pakistan. ${ }^{2}$ Department \\ of Pharmacology, Faculty of Pharmacy, Hamdard University, Karachi.Pakistan.
}

\section{Abstract}

With the advent of the development of biosensor devices, scientists are more focused on emerging new biosensor technologies and tools. The existing application of these devices in fields of biomedicine, drug discovery, food safety, and environmental monitoring get remarkable importance. Till today diagnostic tests especially for early detection and monitoring of cancer and anticancer chemotherapeutic agents bearing major challenges despite their simplicity. This review aims to highlight the use of biosensors as diagnostic means along with their future applicability. In the past various achievement in the development of biosensors opened a new gateway for researchers to further modify biosensing elements to enhance their detection ability particularly in cancer that requires continued development and validation of biomarkers and development of ligands for those biomarkers, as well as continued development of sample preparation methods and multichannel biosensors able to analyze many cancer markers simultaneously. The future for biosensor is promising and bright however require a determined, cost-effective and multi-disciplinary approach to bring out biosensor system from research and development laboratory to market place.

Keywords: Cancer, Biomedicine, Biosensor, Cost-effective, Biomarkers

\section{INTRODUCTION}

After the revolutionary development of the first biosensor device for glucose testing by pioneers Lyon and Clark in 1962 ,there is the growth of demand in the field of medicine and nanotechnology [1]. Attention is being given by researchers to the development of fast and rapid techniques which must be user-friendly and cost-effective. It is assumed that these criteria can be fulfilled by emerging biosensor technologies that require chemistry, nanotechnology, and biological sciences for their fabrication. Although biosensor technology has been applied in numerous medical diagnostics and industries like food, beverages, environmental, agricultural, and many other biotechnological industries [2]. The blood glucose monitoring biosensor device got so much popularity in the market with good precision to measure disease-specific biomarkers both in-vitro and in-vivo [3]. The word "sensor" is a Latin word "sentire" which means "to identify" and this term was first time used by Cammann [4]. The working principle of the biosensor is receptor-transducer based which can interpret the biophysical or biochemical property of the medium. Selectivity and sensitivity are its most captivating character which enable it to detect particular biological molecules in the medium [5].

Various types of biosensors are available like cell-based or microbe-based, enzyme-based, electrochemical, immunesensor, protein, genetically encoded biosensors or DNA, tissue-based, magnetic, optical, piezoelectric biosensors, and thermal. All these biosensors are unique in their manufacturing and application. However, the basic principle for the function of all these biosensors is sample analysis and its recognition, transduction, and amplification. Despite that, all these devices open a new era in the field of medical sciences, and their capability to detect even very minute quantities of the sample increases their demand day by day [6].

Biosensor devices are up-till now applied in various fields and industries. In the medical field, they can be used for the detection and identification of tumors, various pathogens, and their toxins and measurement of blood glucose concentration in diabetic patients. A very useful application of biosensor is the quantitative measurement of the cardiac marker in serum (undiluted), immune-sensor for detection of leukemias, and most recent is quick detection of cancer

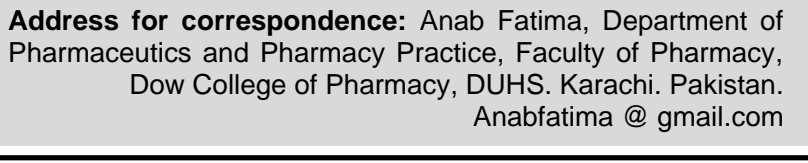

This is an open-access article distributed under the terms of the Creative Commons Attribution-Non Commercial-Share Alike 3.0 License, which allows others to remix, tweak, and build upon the work non commercially, as long as the author is credited and the new creations are licensed under the identical terms.

How to cite this article: Fatima A, Younas I, Ali M W. An Overview on Recent Advances in Biosensor Technology and its Future Application. Arch. Pharm. Pract. 2022;13(1):510. https://doi.org/10.51847/LToGI43jil 
markers by incorporation of the biochip. Diamond microneedle electrode is another innovation used for neurochemical detection [7].

Biosensors have marvelous applications in the food industry for detecting spoiled food gases, identifying contaminated food, and checking the presence and growth of microorganisms i.e. bacteria and fungus in fresh food [8]. In the current scenario of the world, we are facing bioterrorism threats i.e. intentional use of biological entities like B.anthracis (Bacillus anthracis), hepatitis C virus, E-bola, SARS (Severe Acute Respiratory Syndrome) virus, etc for causing harm and biosensors successfully employed to detect these attacks before they prevail [9]. Various research tools were employed to improve selectivity, detection limit, and ultra-sensitivity of these instruments to upgrade them from classical electrochemical to optical/visual, glass, silica, and nano-material respectively [10]. By configuring these devices continuous monitoring and process control can be optimized that will replace annoying and time-consuming diagnostic lab analysis with bed-side monitoring of some advantageous crucial parameters.

\section{Materials and Methods}

A huge amount of literature focused on biosensor technology. This review is a very little effort to systematize all efforts done in a manner that could be helpful for future researchers. Such an attempt could be a handful of guidelines for the researchers belonging to the field of Physics, bio-medicine, and chemists for future advancing bio-sensing technology and challenges to them.

\section{Structural Analysis of Biosensor Designing/Mechanism of Biosensor devices}

First-time potentiometric enzyme-based electrode use as a biosensor device for measurement of blood glucose monitoring [1]. In the recent advances, more energy and time consuming for the development of small-sized, multidimensional, and easy-go biosensors [11]. To accomplish this task different types of bio-sensing elements were applied including enzymes, microbes, organelles, tissue, or cells whose sensitivity is based on catalytic reaction or it may be affinity-based elements like antibodies, receptors, and nucleic acid [12].

The mechanism on which biosensor based is coupling mechanism which may be either physical adsorption encapsulation in which attachment of biological/organic element with sensor-based on physical intermolecular forces [13] or either it may be matrix immobilization where any porous substance like gel matrix act as restricting medium for biological element and creates a direct link with the biosensor element [14]. One of the mechanisms in which biological elements directly link to the sensor by forming covalent amalgamates i.e. termed as covalent coupling [15] while in another mechanism physically organic molecule is entrapped in a particular semi-permeable membrane(act as separating phase between the organic element and analyte) which is directly in contact with sensor element [16].

\section{Technical Approaches for Bio-Transducer} Elements

The main strategy to be used in the biosensor is dependent on whether its detection is labeled or label-free. The labeled detection highly depends on the nature of the label compound to detect the target, such labeled biosensors required specific sensing elements fabricated with immobilized target protein for more reliable detection.

In the case of the label-free method, non-labeled target molecules are detected even which are difficult to tag. Such an approach opens the gateway for interdisciplinary workup of biotechnology with electrical, bio-engineering and electronic engineering to develop label-free biosensors along with a wide range of applications in the environmental and medical sciences [17-19].

\section{Types of Bio-sensors}

The most applicable and common biosensors are electrochemical, optical, and piezoelectrical types. All of these have a common working principle i.e. identification of stimulus released after interaction of the analyte with biosensing element and transform it into a detectable signal (Figure 1).

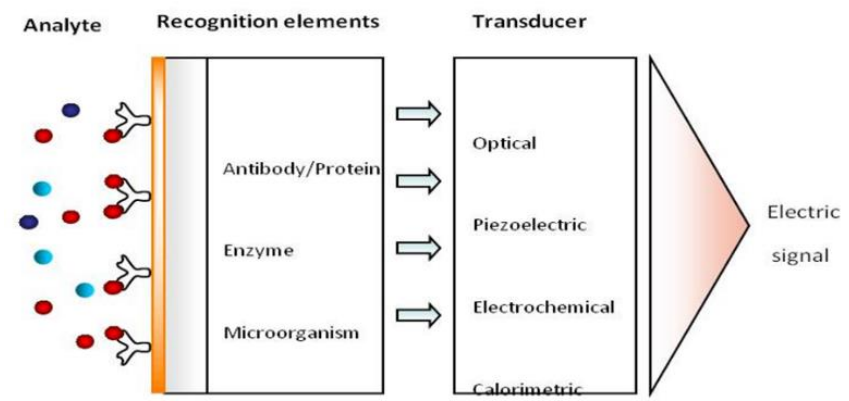

Figure 1. Schematic of a biosensor. (Source: https://yildizuludag.wordpress.com/2009) 
Lyon in 1962. The Glucose biosensors are essential for routine monitoring of blood glucose in diabetic patients widely used in hospitals and clinics. The drawback associated with glucose biosensors is unstable enzyme activity and or inhomogeneity which required further calibration [20]. Currently prepared electrochemical biosensors modified the surface of metal and carbon electrodes by using different biomaterials like enzymes, DNA, or antibody. These biomaterials generate an output signal over the surface of the electrode after specific binding or catalytic reaction. Based on selectivity and stability of differential electrodes and their usage various kinds of electrochemical biosensor has been innovated. The glucose biosensor itself has undergone modification [21]. The principle of electrochemical biosensor has been applied to check the level of antioxidants and reactive oxygen species in the body by the help of detection of Uric Acid in the human physiological system. However, due to the resemblance of Uric acid with oxidation of ascorbic acid, it was no more applicable, and to overcome this innovators modify it into an amperometric detection-based biosensor to quantify both oxidation and reduction potential [22]. To develop a cost-effective and reproducible procedure, the enzymes were immobilized on the surface of electrodes or nano-material based electrodes which is selective, sensitive, and cost-effective uric acid biosensor for routine analysis. Similarly in the same way a wireless mouth guard biosensor was developed to detect salivary levels of Uric acid. in the mouth [23]. Also, electrochemical biosensors are employed for the measurement of hormones although it needs further advancement [24]. Another emerging technology in biosensors is the detection of nucleic acid particularly for gene therapy in genetic disorders [25]. With the advent of recent approaches tobio-fabrication different electrochemical-based biosensors have been modified to promote their use in the field of biomedicine.

\section{Optical/Visual Biosensors}

The mechanism of optical/visual biosensors is based on the measurement of changes in a specific wavelength of light. Its application in the field of biomedicine and environmental need advancement in these biosensors to be simple and ultra-sensitive. By incorporation of dot nano-particles of gold, silica, quartz, glass, or carbon-based materials with micro-fabrication provide a new tool.Highly sensitive and portable Cytochrome P450 enzyme biosensors were developed by using this technology. Similarly, fiber optic chemical biosensors have been employed in the field of biomedicine, drug discovery, and biosensing. Currently, hydrogels are used as entrapment material for sensing elements immobilization. They act as a simple substrate for the immobilization of DNA with the benefits ofentrapment, enhancement of analyte, controlled release, and protection of DNA.Moreover good optical transparency of hydrogels further made a convenient visual detection [26-34].

Silica/Quartz/Crystal and Glass Biosensors
Due to the uniqueness of silica,quartz or crystal and glass recently use in biosensors development.Among them, silicone is more abundantly use because of its optical, mechanical properties, biocompatibility, andelectronic, and more importantly due to its non-toxic nature. The nanomaterial of silicone is employed in a wide range of bioimaging, biosensing, and cancer therapy [33, 34]. The bio-markers that need to be recognized in cancer are shown in Table 1.

\begin{tabular}{|c|c|}
\hline Types of Cancer & Biomarker for detection \\
\hline Breast & $\begin{array}{c}\text { BRCA1, BRCA2, CA 15-3, CA 125, CA } \\
\text { 27.29, CeA, NY-BR-1, iNG-1, HeR2/NeU, } \\
\text { eR/PR }\end{array}$ \\
\hline Colon & $\mathrm{CeA}, \mathrm{eGF}, \mathrm{p} 53$ \\
\hline Esophageal & SCC \\
\hline Liver & $\mathrm{AFP}, \mathrm{CeA}$ \\
\hline Lung & CeA, CA 19-9, SCC, NSe, NY-eSO-1 \\
\hline Melonama & Tyrosinase, NY-eSO-1 \\
\hline Ovarian & $\begin{array}{l}\text { CA 125, HCG, p53, CeA, CA 549, CASA, } \\
\text { CA 19-9, CA 15-3, MCA, MOv-1, TAG72 }\end{array}$ \\
\hline Prostate & PSA \\
\hline
\end{tabular}

Although silicone nano-particles have lots of advantages, some future challenges need to be evaluated such as the method of large-scale development, bio-compatibility, and low-cost production after being marketed. Once addressed these issues will open a new era for silicone nano-material to be used as biosensor material [26, 28-30, 34].

\section{Bio-Sensors based on Nano-Materials}

A variety of nano-particles are used for biosensor immobilization. They include Gold, silicone,silver,copper nanoparticles, and carbon-based resources like graphite, grapheme and carbon nanotubes, etc [18, 27, 34-40]. Due to the highly sensitive and specific nature of these nanoparticles, the biosensors that were developed based on nanomaterials also have a high degree of specificity and sensitivity. Gold nano-particles are the most stable metallic nano-material against oxidation with no internal toxicity [41]. Unlike silver which undergone oxidation with the potential of toxicity when administered internally [42]. Despite these facts, nan-materials are considered a good source to enhance the detection and sensitivity of biosensor devices for single-molecule detection [19]. One of the examples is platinum-based nano-particles use for the detection of low concentrations of DNA [30]. In the same way, quantum dots and iron oxide nano-crystals effectively target tumor antigens [43].

\section{Synthetic Fluorescent / Genetically Encoded Biosensors}

Such types of biosensors open a way of understanding the biological process as well as molecular pathway inside the 
cell [44-46]. Initially, fluorescently tagged antibodies were developed for imaging of fixed cells. This idea leads to the development of sensors using second messengers, small molecule binding to analytes, and biological proteins [46]. Currently, fluorescent biosensors analyzing motor protein with single-molecule detection [45]. However, it's a difficult task along with all advantages. Those genetically encoded biosensors which target those components involved in energy production provide an opportunity to be aware of mitochondrial functioning. These components include reactive oxygen species and cAMP [47]. Similarly, FRETbased biosensors (Förster resonance energy transfer) were developed for imaging of cAMP, cGMP, and calcium in the cells among which cGMP is a specific signaling molecule in the cardiovascular system [48]. With the help of process optimization, these approaches have been used to develop various microbial and cell organelles-based biosensors with a particular target $[42,46]$. Even though they are much better detected by electrochemical, electromechanical, and optical biosensors than any molecular technique [49]. At the time this field requires to move toward the whole genome with optical-based genetic biosensors. It has been admitted that such a goal could be achieved by optical-based biosensors with small molecules /nanomaterials and fluorescence combinations.

\section{Microbial Biosensors}

The innovative technologies recently employed for environmental monitoring and bioremediation. Genetic/protein engineering and synthetic biology-based technologies are used for programming microorganisms that are specific and sensitive, producing specific signal output. Such biosensors have application in the field of bioremediation where live cells having enzyme activity degrade xenobiotics [50]. Similarly, biochemical oxygen demand and toxicity in the environment can be monitor by newly developed microbial fuel-based biosensors. The bacteria can utilize organic substrate and convert it into electrical energy for fermentation. Such bacterial potential use by bio-electrochemical devices that control microbial respiration that convert organic substrate to electrical energy. Along with these novelties considering production and operating cost, there are certain limitations with microbial biosensors because of low power density. A lot of work has been done to incorporate new systemic approaches to enhance the performance and to cut down the cost [10]. Microbial biosensors are also applied for pesticide and heavy metal detection with a preference over eukaryote micro-organisms than on eukaryote. This is due to the wider sensitivity of higher eukaryotic microbes to different toxic molecules and has relevance to higher animals [51]. The microbial biosensor's future is promising with its application in environmental pollution monitoring and sustained energy production $[10,52]$.

\section{Results and Discussion}

Different types of biosensors and their application has been discussed previously. In this section assessment of different types of biosensors in terms of their selectivity, specificity, sensitivity, time, cost and portability will be discussed.

The development of electrochemical sensors lead it to a large-scale consumer market for glucose and pregnancy test due to its low cost focusing on portability, analysis time, and detection limit [19]. The incorporation of polymers and nano-materials for immobilization of analytes are the key components to enhance the detection limit and sensitivity of biosensors. Such a technique generates the bio-fabrication idea using either contact or non-contact-based patterning. By applying lateral flow technology the samples can be directly delivered to the desired target by creating specific direct interaction. Gold, silver, and silicone-based biofabrication gave new methods to develop electrochemical sensors with high sensitivity and specificity with real-time analysis. But their regenerative ability is marked as their limitation for long-term use. A very advantageous method is single analyte detection with contact-based sensing. To achieve this transducers based on FRET, fluorescent-based, surface Plasmon, and bioluminescent resonance energy transfer have been employed [28] which improve singlemolecule detection but no application on multiple detections. To detect multiple analytes micro or nano cantilever transducer in electrochemical sensor biofabrication has wider use. Also, non-contact-based sensors with laser direct or 3D bioprinting have got good achievements but the major barrier in their success is high cost and customization. Notwithstanding some amperometric electrochemical biosensors have been developed [23]. They use body fluids for disease diagnosis. Another innovation in the field of biosensors is fiber-optic chemistry-based Optic biosensors and they are excellent in single-molecule detection like DNA or peptide. Preferably they have application in the field of biomedicine and forensic sciences. By combining different materials like enzyme/substrate, antigen/antibody, or nucleic acid brought insurgency in the technology of optical biosensors. Similarly, there is also the option to incorporate sections of tissues, plant or animal cells, microorganismsin the biosensing system. In practice, optical biometric recognition systems are offered by molecular optoelectronics. Due to recent advances in surface analysis morphology with the help of high-end electrons and atomic force microscopy the optical biosensor technology get so much improvement. However, their detection limit had not been closer to Femtolevel. Currently, in the modern optic technology, using nano-mechanical bio-sensors based on surface resonance technology and microcantilevers invented DNA chip for sensitive analysis and real time-specific [53-55]. Optical biosensors have the advantage of providing speedy swift analysis and capability to provide a spectrum of information but with the limitation of its high cost and with the technical lissues like intricacy in immobilization for bio-fabrication and the sterilized environment requirement. These problems are required to be addressed to get optimal advantages from optical biosensors. 
The technique of bio-fabrication provides better results for mass-based biosensors. Along with the advancement in micro and nanofabrication mechanical devices with nanosized moving parts have been established. The fabrication principle bridges the principle of biophysics and bioengineering to develop practical micro and nanoelectromechanical biosensors. At the time there are so many challenges in mass-based sensors for better-capturing agents to fabricate at nano-scale with the help of microelectronic fabrication. The application potential of semiconductor materials and quantum dot technology is worth mentioning here. Till now, there is room for improvement in the biosensor technology for large arrays in real-time, quantitative assays. The use of micro-and nano scale cantilevers fabrication might be promising [35]. Genetically encoded or synthetic fluorescent biosensors area major technical innovation. They analyze the molecular mechanism of biological processes [44-46]. Although these biosensors got a tremendous future appreciation for singlemolecule detection, they require outstanding instrumentation for methodology, probe preparation, and detection which in practice are much difficult. Due to high sensitivity and selectivity, microbial fuel-based biosensors have got dissimilarities in bioremediation but they involve high cost and complex procedures to develop microbial strains. It involves complex genetic engineering methods and mass production of microbial strains with strict governing laws and ethical clearance in addition to production cost management. They are good bioremediation tools for environmental monitoring.

Taking in view by utilizing optic bio-electronic or electrochemical principles with a combination of biological materials or biomolecules, nanomaterials, and polymers different integrated technologies are involved to develop different micro and nano biosensor platforms to attain highly sensitive devices [55].

A wide range of technologies including electrochemical, electromechanical, and fluorescence- cum-optical-based biosensors and genetically engineered microbes are integrated into the modern methods for biosensor development. In disease diagnosis and therapy, some of them have remarkable application. Currently, there is a strong need forbio-fabrication with increasing demand for rapid analyses with the cost-effectiveness that bridges a way to identify cellular to whole animal activity with a detection limit of high accuracy for single molecules. In the scenario where biosensors work under multiplex conditions, 2D and 3D detection are employed with sophisticated transducers for targeting and quantifying small analytes of interest [28]. Several innovations have been made for this purpose at different levels. The future target is to develop a more robust regenerative biosensor for long-term application. Once achieved this will be a helpful tool for clinicians and patients for the effective diagnostic and therapeutic use of biosensors. The use of nanomaterials in the form of quantum dots plays a pivotal role by applying nanotechnology.In this receptor, elements are used not only to detect different types of tumors but also to deliver drugs to the tumor. There are so many hurdles and once they will overcome then it will be a great achievement in the history of mankind. It needs high specificity, selectivity with minimum cost and time. In the past various achievement in the development of biosensors opened a new gateway for researchers to further modify biosensing elements to enhance their detection ability particularly in cancer that requires continued development and validation of biomarkers and development of ligands for those biomarkers, as well as continued development of sample preparation methods and multi-channel biosensors able to analyze many cancer markers simultaneously.

\section{ConcLusion}

The future for biosensor is promising and bright however require a determined, cost-effective and multi-disciplinary approach to bring out biosensor system from research and development laboratory to market place. The main key for the development of a successful biosensor involves a better combination of bio-sensing and bio-fabrication with synthetic biological approaches using either electrochemical or bio-electronic principles or optic. Continuous research will be helpful to overcome all challenges with the application of biosensors in cancer.

ACKNOWLEDGMENTS: I hereby thank my co-authors who contributed a lot in completing this review.

CONFLICT OF INTEREST: None

FINANCIAL SUPPORT: None

ETHICS STATEMENT: None

\section{REFERENCES}

1. Clark Jr LC, Lyons C. Electrode systems for continuous monitoring in cardiovascular surgery. Ann N Y Acad Sci. 1962;102(1):29-45.

2. Nurunnabi M, Cho KJ, Choi JS, Huh KM, Lee YK. Targeted near-IR QDs-loaded micelles for cancer therapy and imaging. Biomaterials. 2010;31(20):5436-44.

3. Malima A, Siavoshi S, Musacchio T, Upponi J, Yilmaz C, Somu S, et al. Highly sensitive microscale in vivo sensor enabled by electrophoretic assembly of nanoparticles for multiple biomarker detection. Lab Chip. 2012;12(22):4748-54.

4. Cammann K. Bio-sensors based on ion-selective electrodes. Fresenius Z Anal Chem. 1977;287(1):1-9.

5. Wang JX, Sun XW, Wei A, Lei Y, Cai XP, Li CM, et al. Zinc oxide nanocomb biosensor for glucose detection. Appl Phys Lett. 2006;88(23):233106.

6. Giepmans BN, Adams SR, Ellisman MH, Tsien RY. The fluorescent toolbox for assessing protein location and function. Science. 2006;312(5771):217-24.

7. Situ C, Mooney MH, Elliott CT, Buijs J. Advances in surface plasmon resonance biosensor technology towards high-throughput, food-safety analysis. Trends Analyt Chem. 2010;29(11):1305-15.

8. Tamayo J, Humphris AD, Malloy AM, Miles MJ. Chemical sensors and biosensors in liquid environment based on microcantilevers with amplified quality factor. Ultramicroscopy. 2001;86(1-2):167-73.

9. Edelstein RL, Tamanaha CR, Sheehan PE, Miller MM, Baselt DR, Whitman L, et al. The BARC biosensor applied to the detection of biological warfare agents. Biosens Bioelectron. 2000;14(10-11):80513.

10. Du Z, Li H, Gu T. A state of the art review on microbial fuel cells: a promising technology for wastewater treatment and bioenergy. Biotechnol Adv. 2007;25(5):464-82. doi:10.1016/j.biotechadv.2007.05.004 
Fatima et al:: An Overview on Recent Advances in Biosensor Technology and its Future Application-A Review

11. Chao J, Zhu D, Zhang Y, Wang L, Fan C. DNA nanotechnologyenabled biosensors. Biosens Bioelectron. 2016;76:68-79.

12. Hasan A, Nurunnabi M, Morshed M, Paul A, Polini A, Kuila T, et al. Recent advances in application of biosensors in tissue engineering. BioMed Res Int. 2014;2014.

13. Wang J, Musameh M. Carbon-nanotubes doped polypyrrole glucose biosensor. Anal Chim Acta. 2005;539(1-2):209-13.

14. Gupta R, Chaudhury NK. Entrapment of biomolecules in sol-gel matrix for applications in biosensors: Problems and future prospects. Biosens Bioelectron. 2007;22(11):2387-99.

15. Bisht V, Takashima W, Kaneto K. An amperometric urea biosensor based on covalent immobilization of urease onto an electrochemically prepared copolymer poly (N-3-aminopropyl pyrrole-co-pyrrole) film. Biomaterials. 2005;26(17):3683-90.

16. Doretti L, Ferrara D, Lora S, Schiavon F, Veronese FM. Acetylcholine biosensor involving entrapment of acetylcholinesterase and poly (ethylene glycol)-modified choline oxidase in a poly (vinyl alcohol) cryogel membrane. Enzyme Microb Technol. 2000;27(3-5):279-85

17. Citartan M, Gopinath SC, Tominaga J, Tang TH. Label-free methods of reporting biomolecular interactions by optical biosensors. Analyst. 2013;138(13):3576-92. doi:10.1039/c3an36828a

18. Sang S, Wang Y, Feng Q, Wei Y, Ji J, Zhang W. Progress of new label-free techniques for biosensors: a review. Crit Rev Biotechnol. 2016;36(3):465-81. doi:10.3109/07388551.2014.991270

19. Turner AP. Biosensors: sense and sensibility. Chem Soc Rev. 2013;42(8):3184-96. doi:10.1039/c3cs35528d

20. Harris JM, Reyes C, Lopez GP. Common causes of glucose oxidase instability in in vivo biosensing: a brief review. J Diabetes Sci Technol. 2013;7(4):1030-8.

21. Wang B, Takahashi S, Du X, Anzai JI. Electrochemical biosensors based on ferroceneboronic acid and its derivatives: a review. Biosensors. 2014;4(3):243-56.

22. Erden PE, Kılıç E. A review of enzymatic uric acid biosensors based on amperometric detection. Talanta. 2013;107:312-23. doi:10.1016/j. talanta.2013.01.043

23. Kim J, Imani S, de Araujo WR, Warchall J, Valdés-Ramírez G, Paixão TR, et al. Wearable salivary uric acid mouthguard biosensor with integrated wireless electronics. Biosens Bioelectron. 2015;74:1061-8. doi:10.1016/j.

24. Bahadır EB, Sezgintürk MK. Electrochemical biosensors for hormone analyses. Biosens Bioelectron. 2015;68:62-71. doi:10.1016/j.bios.2014.12.054

25. Hamidi-Asl E, Palchetti I, Hasheminejad E, Mascini M. A review on the electrochemical biosensors for determination of microRNAs. Talanta. 2013;115:74-83. doi:10.1016/j.

26. Dias AD, Kingsley DM, Corr DT. Recent advances in bioprinting and applications for biosensing. Biosensors. 2014;4(2):111-36. doi: $10.3390 /$ bios402011

27. Guo X. Single-molecule electrical biosensors based on single-walled carbon nanotubes. Adv Mater. 2013;25(25):3397-408. doi:10.1002/ adma.201301219

28. Khimji I, Kelly EY, Helwa Y, Hoang M, Liu J. Visual optical biosensors based on DNA-functionalized polyacrylamide hydrogels. Methods. 2013;64(3):292-8. doi:10.1016/j

29. Kwon SJ, Bard AJ. DNA analysis by application of Pt nanoparticle electrochemical amplification with single label response. J Am Chem Soc. 2012;134(26):10777-9. doi:10.1021/ja304074f

30. Li M, Li R, Li CM, Wu N. Electrochemical and optical biosensors based on nanomaterials and nanostructures: a review. Front Biosci (Schol Ed). 2011;3:1308-31. doi:10.2741/228

31. Ogi H. Wireless-electrodeless quartz-crystal-microbalance biosensors for studying interactions among biomolecules: A review. Proc Jpn Acad Ser B. 2013;89(9):401-17. doi:10.2183/pjab.89.401

32. Peng F, Su Y, Zhong Y, Fan C, Lee ST, He Y. Silicon nanomaterials platform for bioimaging, biosensing, and cancer therapy. Acc Chem Res. 2014;47(2):612-23. doi:10.1021/ar400221g

33. Shen MY, Li BR, Li YK. Silicon nanowire field-effect-transistor based biosensors: From sensitive to ultra-sensitive. Biosens Bioelectron. 2014;60:101-11. doi:10.1016/j.
34. Schneider E, Clark DS. Cytochrome P450 (CYP) enzymes and the development of CYP biosensors. Biosens Bioelectron. 2013;39(1):1 13. doi:10.1016/j.

35. Zhou Y, Chiu CW, Liang H. Interfacial structures and properties of organic materials for biosensors: An overview. Sensors. 2012;12(11):15036-62. doi:10.3390/s121115036

36. Hutter E, Maysinger D. Gold-nanoparticle-based biosensors for detection of enzyme activity. Trends Pharmacol Sci. 2013;34(9):497507. doi:10.1016/j.

37. Ko PJ, Ishikawa R, Sohn H, Sandhu A. Porous silicon platform for optical detection of functionalized magnetic particles biosensing. J Nanosci Nanotechnol. 2013;13(4):2451-60. doi:10.1166/jnn.

38. Lamprecht C, Hinterdorfer P, Ebner A. Applications of biosensing atomic force microscopy in monitoring drug and nanoparticle delivery. Expert Opin Drug Deliv. 2014;11(8):1237-53.

39. Senveli SU, Tigli O. Biosensors in the small scale: methods and technology trends. IET Nanobiotechnol. 2013;7(1):7-21. doi:10.1049/ iet-nbt.2012.0005

40. Valentini F, Fernàndez LG, Tamburri E, Palleschi G. Single walled carbon nanotubes/polypyrrole-GOx composite films to modify gold microelectrodes for glucose biosensors: study of the extended linearity. Biosens Bioelectron. 2013;43:75-8. doi:10.1016/j.bios.2012.11.019

41. Su L, Jia W, Hou C, Lei Y. Microbial biosensors: a review. Biosens Bioelectron. 2011;26(5):1788-99. doi:10.1016/j.bios.2010.09.005

42. Nie S, Xing Y, Kim GJ, Simons JW. Nanotechnology applications in cancer. Annu Rev Biomed Eng. 2007;9:257-88. doi:10.1146/annurev. bioeng.9.060906.152025

43. Kunzelmann S, Solscheid C, Webb MR. Fluorescent biosensors: design and application to motor proteins. Fluorescent Methods for Molecular Motors. 2014:25-47. doi:10.1007/978-3-0348-0856-9_2

44. De Michele R, Carimi F, Frommer WB. Mitochondrial biosensors. Int J Biochem Cell Biol. 2014;48:39-44. doi:10.1016/j.biocel.2013.12.014

45. Oldach L, Zhang J. Genetically encoded fluorescent biosensors for live-cell visualization of protein phosphorylation. Chem Biol. 2014;21(2):186-97. doi:10.1016/j.

46. Randriamampita C, Lellouch AC. Imaging early signaling events in $\mathrm{T}$ lymphocytes with fluorescent biosensors. Biotechnol J. 2014;9(2):203-12. doi:10.1002/biot.201300195

47. Johnson BN, Mutharasan R. Biosensor-based microRNA detection: techniques, design, performance, and challenges. Analyst. 2014;139(7):1576-88. doi:10.1039/c3an01677c

48. Park K, Jung J, Son J, Kim SH, Chung BH. Anchoring foreign substances on live cell surfaces using Sortase A specific binding peptide. Chem Commun. 2013;49(83):9585-7. doi:10.1039/c3cc44753g

49. Gutiérrez JC, Amaro F, Martín-González A. Heavy metal whole-cell biosensors using eukaryotic microorganisms: an updated critical review. Front Microbiol. 2015;6:48. doi:10.3389/fmicb.00048

50. Sun JZ, Peter Kingori G, Si RW, Zhai DD, Liao ZH, Sun DZ, et al. Microbial fuel cell-based biosensors for environmental monitoring: a review. Water Sci Technol. 2015;71(6):801-9. doi:10.2166/wst.2015.035

51. Scheller FW, Yarman A, Bachmann T, Hirsch T, Kubick S, Renneberg R, et al. Future of biosensors: a personal view. Adv Biochem Eng Biotechnol. 2014;140:1-28. doi:10.1007/10_2013_251

52. Wang S, Poon GM, Wilson WD. Quantitative investigation of protein-nucleic acid interactions by biosensor surface plasmon resonance. Methods Mol Biol. 2015;1334:313-32. doi:10.1007/9781-4939-2877-4_20

53. Arlett JL, Myers EB, Roukes ML. Comparative advantages of mechanical biosensors. Nat Nanotechnol. 2011;6(4):203-15. doi:10.1038/ nnano.2011.44

54. Tothill IE. Biosensors for cancer markers diagnosis. Semin Cell Dev Biol. 2009;20(1):55-62.

55. Zhang Z, Liu J, Qi ZM, Lu DF. In situ study of self-assembled nanocomposite films by spectral SPR sensor. Mater Sci Eng C. 2015;51:242-7. doi:10.1016/j.msec.2015.02.026 